numériques. Les observations d'une année relatives à une même station seront groupées ensemble dans un seul tableau numérique dans lequel les résultats mensuels seront disposés en colonnes verticales et les observations journalières en lignes horizontales. A chaque jour correspondront deux lignes: sur la première sera inscrite, en bleu, la cote de la hauteur de l'eau, sur la seconde, en noir, des qu'on possèdera les données nécessaires, le débit correspondant. Ces tableaux seront imprimés d'un seul côté de la feuille, de telle façon qu'on puisse, pour l'étude détaillée d'une station ajouter les uns aux autres les tableaux de plusieurs années successives, et en juxtaposant les colonnes d'un même mois, faire les moyennes de ce mois, etc.

Les graphiques seront également tracés d'un seul côté de la feuille et disposés de façon qu'on puisse, pour l'étude d'une station, ajouter sans solution de continuité une série d'années. Les mèmes graphiques pourront, par une simple transposition d'échelles des ordonnées, servir à représenter solt des hauteurs d'eau, soit des débits; à cet effet les deux échelles seront indiquées, la première en bleu et la seconde en noir. Les jaugeages opérés dans l'aunée seront marqués d'un point noir placé sur le graphique et cotés parlavaleur, chiffrée en noir, du débit trouvé.

Chaque publication annuelle débutera par une liste des stations hydrométriques et des stations de jaugeage avec les noms des observateurs et des opérateurs. Elle se terminerapar les remarques utiles concernant les observations et les jaugeages, l'ensemble de ces remarques constituant un compte rendu annuel. Au début, les tableaux numériques et les graphiques ne seront dressés que pour les stations les plus importantes: celles qui paraitront selon toutes probabilités devoir se prêter à des observations régulières et continues. Leur nombre, petit au début, augmentera peu à peu. Il ne sera pas publié avec trop de hâte les résultats de stations qui pourraient ensuite être abandonnées.

Dans l'ensemble des postes, il en sera donc choisi, par une première sélection, un certain nombre dont les résultats seront publiés chaque année comme il vient d'être dit. Ces postes, une fois choisis, continueront à figurer dans la publication et leur nombre s'en accroîtra insensiblement.

Par une deuxième sélection il sera choisi tous les ans quelques postes, pour lesquels on possèdera les données suffisantes, afin d'y représenter avec précision le régime du cours d'eau au moyen des éléments que voici :

a) D'abord un croquis de la station avec la position des échelles de repères, etc, et l'emplacement des jaugeages, profils en long et en travers. S'il s'agit d'un lit mobile, seront indiquées les modifications successives correspondant aux diverses époques où l'on a effectué des relevés sur le terrain.

b) En second lieu, la formule servant à transformer les hauteurs d'eau en débits, ou, s'il s'agit d'un lit mobile, les diverses valeurs des cofficients de la formule correspondant aux divers états du lit. Cette formule sera traduite sous deux formes: celle d'un tableau numérique et celle d'une courbe algébrique dite "caractéristique ».

c) En troisième lieu, la courbe de régime à la station considérée, la courbe des débits mensuels moyens avec l'enveloppe des débits extrêmes. Ces diverses courbes tracées à l'aide de résultats d'expériences d'un certain nombre dannées, permettront de dégager les deux débits caractéristiques - dont il a été question au début - qui doivent servir à mesurer la puissance hydraulique.
A partir du moment ou auront été publiées, pour une station déterminée, les trois catégories de renseignements $a, b, c$, on pourra considérer comme bien connu le régime actuel du cours d'eau en ce point, et comme terminée l'œuvre du recensement de sa puissance hydraulique. La continuation de la publication des observations hydrométriques n'aura plus d'autre objet que de permettre de suivre les transformations du régime et de constater les modifications que subit arec le temps la puissance hydraulique.

B.-Dans la catégorie des publications définitives faites une fois pour toutes figureront:

1 Le tableau des surfaces des bassins décomposés par altitudes et par nature de terrains. Ce tableau sera accompagné d'une carte de délimitation des bassins, sur laquelle les emplacements des stations hydrométriques et pluviométriques seront indiquées et les terrains distingués par degré d'imperméabilité.

$2^{\circ}$ Le profil en long des cours d'eau, accompagné de la liste des repères qui auront été posés le long de ces cours d'eau par le service du nivellement général de la France et répertoriés par les soins de ce même service.

$3^{\circ}$ Le tableau des puissances hydrauliques des divers tronçons des cours d'eau, dressé d'après les résultats consignés au "tableau des surfaces» et auquel sera joint une carte schématique des puissances, analogue à celles qui ont été dressées par les ingénieurs des Hautes-Alpes pour l'Fxposition de igoo et par l'ingénieur en chef de la Savoie.

Lorsque l'ensemble de ces trois publications relatives à une vallée sera terminé, il suffira d'y ajouter les observation des pluies, neiges, glaciers et sources, publiées ailleurs, pour grouper les éléments essentiels d'une monographie hydrologique de la vallée répondant parfaitement à l'objet du Service d'études.

Il n'est pas nécessaire d'attendre jusqu'à ce moment pour comprendre l'utilité que présentera la publication de monographies semblables. Le Service, organisé et dirigé par MM. Tavernier et DE LA Brosse, avec autant de science et d'habileté que de dévouement, lendra à notre inctustrie hydraulique les plus sıgnalés services. Sachons en attendre patiemment les résultats car, comme on a pu s'en convaincre, c'est un travail de très longue haleine.

\section{Recherches sur la synthèse directe de l'acide azotique et des azotates à la température ordinaire}

La combinasson à l'azole avec l'oxygène est actuellement l'uno des question qui passionne le plus le monde industriel. La fabricalion des nilrates apparaissant comme l'un des plus imporlants cóbouchés de l'industre hydro-clectrique, les lecleurs de $L a$ Houille Blanche liront avec inlérêl la communicalion sulvanle qui a élé faite par M. Bertherot, à l'Académie des Sciences, dans sa séance du 18 juin 1906 , el que nous reproduisons in-exlenso.

J'ai entrepris de nouvelles recherches sur la synthèse de l'acide azotique par les éléments, à la température ordinaire, sous l'influence de l'effluve électrique. J'ai reconnu que cette synthèse peut être effectuée d'une façon directe et intégrale, dans des conditions que j'ai découvertes et que je vais décrire, et cela sans complications ni formations secondaires. Ces résultats distinguent les expériences que je vais décrire dés réactions connues: opérées avec l'étincelle électrique (ou avec l'arc) dans l'expérience classique de Gavendish; aussi bien dans l'oxydation partielle de l'azote, 
au cours des combustions simultanées du carbone, du soufre. de l'hydrogène et autres corps inflammables $\left(^{\star}\right)$; ou bien encore, dans les essais exécutés juscu'ici par différentes autres voies, telles que la nitrification naturelle, accomplie avec le concours préalable des agents microbiens fixateurs d'azote $\left(^{\star *}\right)$.

Je vais résumer mes études sur la formation de l'acide azotique par l'azote et l'oxygène, à la température ordinaire: en en fixant les proportions relatives, en présence de l'eau ou des alcalis, et en déterminant les limites de la combinaison et sa vitesse, suivant l'élat de condensation de la matière, c'est-á-dire suivant la pression des systèmes gazeux. Je terminerai par des considérations thermochimiques, destinées à comparer la mêthode actuelle de synthèse avec les autres méthodes, fondées sur l'emploi de l'étincelle, ou des combustions simultanées.

\section{I. - Méthode}

J'opère au moyen des appareils que j'ai imaginés pour faire agir l'effuve, soit sur un courant gazeux, soit sur un volume déterminé de gaz rentermé dans un vase de verre scellé, en présence de l'eau ou d'une solution étendue de potasse. Dans plusieurs cas, ce vase a été relié à un tube vertical capillaire, immergé dans une cuve à mercure: de facon à pouvoir sujvre la marche des pressions, et par conséquent la marche de la combinaison à volume constant, accomplie au cours d'intervalles de temps successils. Le vase était entouré d'eau, définissant approximativement la température des gaz, maintenue à peu près constante, depuis $8^{\circ}$ jusqu'à $80^{\circ}$, dans différentes expériences.

Au cours de tous mes essais, il se forme uniquement, ou à peu près, de l'acicle azotique hydraté, d'après la formule exacte:

$$
\mathrm{Az}^{2}+\mathrm{O}^{5}+\mathrm{H}^{2} \mathrm{O}+\mathrm{Aq}=2 \mathrm{AzO}^{3} \mathrm{H} \text { étendu }
$$

cet acide demeurant libre, ou combiné à la potasse.

La réaction, à chacque instant, s'effectue suivant cette formule; quel que soit l'excès relatif de l'azote, sans qu'il se forme ni acide azoteux bien sensible, ni ammoniaque.

De même, avec un excès d'oxygène.

Dans tous les cas, elle s'accomplit jusqu'á épuisement de celui des deux gaz qui se trouve en excés.

Ces résultats ont été constatés à l'aide de mesures effectuées par les analyses suivantes:

10 Mesure exacte des volumes d'oxygène et d'azote disparus :

$2^{\circ}$ Mesure du titre acide des solutions aqueuses, ou du titre alcalin des solutions potassicues;

3" Mesure du volume de bioxy de d'azote gazeux, régénéré ensuite de l'azotate.

Dans la plupart des expériences, les trois ordres de mesures ont été exécutés sur le mème échantillon et ont été concordantes. En outre, on a toujours vérifié avec précision la dose ou l'absence sensible de l'acide azoteux et l'absence de l'ammoniacue.

L'effuve était produite à l'aide d'une bobine d'induction à décharges alternatives, alimentée par un courant de 12 volts et 6 ampères, d'intensité moyenne (courant primaire). La longueur des étincelles explosives entre les pòles étalt lımitée a $20^{\mathrm{mm}}$. Mais on avait soin de régler l'interrupteur vibrant, de facon à ne produire à l'intérieur des gaz du vase à effluves, ni étincelles, ni pluie de feu. J'ai opéré tantôt sans condensateur, tantôt avec condensateur. Jamais la vapeur nitreuse n'a apparu, même temporairement. La réaction opérée sur un volume limité de gaz avait en général atteint sensiblement sa limite au bout de 6 à 8 heures. () Vorr entr'autres mes expériences Annales de chimie et do Phy
sique, "e sérte, t. XXI. p. $145-202$.

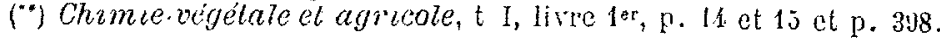

\section{II. - Expériences. Réaction totale. Proportions relatives.}

J'ai opéré à la lempérature ơrdinaire, en faisant varier les proportions relatives, c'est-à-dire en employant l'azote l'oxygène, d'abord suivant les rapports thériques de 2 vo. lumes à 5 volumes: $A z^{2}+O^{5}$; puis suivant les rapporls de 2 volumes à un seul: $A z^{2}+O$; enfin suivant les rappopts de l'air atmosphéricue: $79^{\mathrm{vol}}, 2$ azote $+20^{\mathrm{vol}}, 8$ oxygène, rapports voisins de $A z^{i}+O$.

La vapeur d'eau, présente au cours de mes expériences, était toujours en proportion équivalente fort inférieure celles de l'azote et de l'oxygène lorsque j'ai opéré à la température ordinaire; si ce n'est vers la fin du phénoméne. En effet, au début, vers $8^{\circ}$, le volume de la vapeur d'eau ne représente guère que le centiène des volumes réunis des deux gaz, sous la pression normale, tandis que vers la fin elle peut en constituer la presque totalité, Iorsqu'on opere à volume constant, en partant de $\mathrm{Az}^{2}+\mathrm{O}^{5}$.

Cependant, en raison de la lenteur de la réaction, la vapeur d'eau qu'elle ajssorbe est sans cesse reformée aux dépens de l'eau liquide placée au fond des tubes $\left(^{*}\right)$, et elle est régénérée assez rapidement, de façon à se trouver tou. jours en excès suffisant pour la production contınue de lacide azotique; ainsi que le prouve l'absence perpétuell de la vapeur nitreuse.

On a opéré d'abord par circulation, puis sur un volume de gaz limité, contenu dans un appareil complètement. clos.

\section{Premiere partie. - Circulation}

(1) Air atmosphérique. - J'ai fait circuler lentement un courant d'air dans mon appareil à ozone $\left(^{*}\right)$, à raison d'un demi-litrs par heure, vers $10^{\circ}$, pendant 8 heures. Je donneral comme indication les doses d'acide azotique formé; ces doses étant évidemment subordonnées au volume du ga contenu dans l'espace annulaire compris entre les deu cylindres de verre concentriques, à la distance de ces deux cylindres, et à la tension (variable) de l'électricité fournie à l'effluve par la bobine d'induction.

On a dosé, d'une part, l'acide condensé dans l'eau, alk fond du cylindre enveloppant, et d'autre part, l'acide vapo. risé et entraîné au dehors par l'excès des gaz non combinés, barbotant dans une antre proportion d'eau.

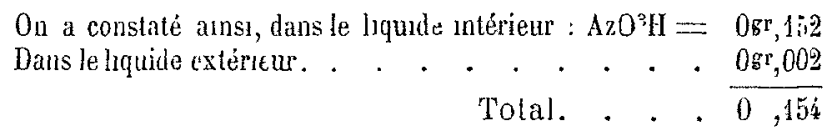

Ce qui répond en moyenne, pour la durée d'une heure, à $0 \mathrm{~g}, 0192$, correspondant à $3 \mathrm{cc}, 6$ d'azote et $12 \mathrm{cc}, 6$ d'oxygène, empruntés à un demi-litre d'air.

On voit, en outre, que la tension de vapeur propre de l'acide (hydraté) est très faible.

$\mathrm{Ni}$ acide azoteux, ni ammoniaque.

(2) Même expérience, avec le même appareil ; à cela prés que l'on y avait introduit une solution étendue de polasse au lieu d'eau. On a fait passer 5 litres d'air, en 8 heures. la fin on a obtenu: 0sr,202 d'acide azotique $\mathrm{AzO}$ FI (neutralisa par la potasse); toujour's sans acide azoteux, nı ammo. niaque.

Or, dans ces conditions, s'il y avait eu de l'acide azoteux, ol du gaz hypoazotique, même temporaircment, il aurait dû sa former, d'après les expériences de Gay-Lussac et les mient nes, de l'azotite, au contact de la potasse; l'acide azoteur étant soustrait soudain au mélange gazeux, avant qu'il ait eu le temps d'ètre suroxydé.

(") L'eau liqude introduite dans l'appareil ne dort jamais atteind même au début, une hauteur suftisante pour établir la conduchblth électrique entre los parors de corre des deux tubes concentriques, enth lesquelles se produrt l'eflure.

(") Annales de Chimie el de Physique, 5e série, t. $\mathrm{x}, \mathrm{p} .166$. 
La dose diacide azotique formé par heure, au cours de cet essai, fait avec le concours de la potasso, était de ngr. (252; soit. 0\%, 0050 par litre d'air employé : chiffre concordant avec celui de l'acide formé a vec le concours de lieau pure $\left(06^{\prime}, 0048\right)$. L'alcali n'avait donc exercé aucune' action aucélératrice sensible sur la combinaison des deux gaz, dans ces conditions.

Ajoutons d'ailleurs que le poids de l'oxygène contenu dans un litre d'air employé était 0rr,027, et celui de l'oxygène changé en acide azotique 0rr,00375. Ces chiftres répondent à un rendement de 14 pour 100 , constaté en présence d'un excès d'oxygène et d'un excès d'azote non combinés.

On va montrer que ce rendement peut devenir total, tant pour l'oxygène que pour l"azote, lorsque l'on prolonge la réaclion su' un volume d'air limité.

(3) Mais avant d'aborder ce sujet, j'ai recherché si la formation de l'acide azotique, sous l'iufiuence de leffluve, eximealt les hautes tensions développées avec la bobine d'induction, mise en jeu dans les conditions précédentes. or, en opérant avec le courant alternatif du secteur, c'esta-dire sous une tension d'une centaine de volts, avec les mêney appareils et avec la mème épaisseur de gaz dans l'espare annulaire qui sépare les deux tumes concentriques, 4 litı'es d'air humide, circulant pendant 8 heures, n'ont toumi aucune traces d'acide azotique.

Dans un appareil clos, en opérant en vase scellé sur un volume limité d'une trentaine de centimètres cubes, pendant 18 heures. le résultat a été également négatif avec le courant du secteur (105 volts) ( $0^{*}$ ).

SECONDE PARTIE. -- EXPÉRIENCES FAITES SUR UN VOLUME CONSTANT DE GAZ, EN VASES CLOS ET SCELLÉS A LA. LAMPE. LIMITE DE LA COMBINAISON.

On a fait varier les proportions retatives des deux gaz, ainsi qu'il a été dít plus haut:

(4) Mélange en propoption théorique: $\mathrm{Az}^{2}+\mathrm{O}^{5}$; en pré-

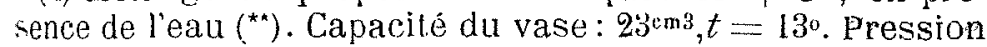
intêrteure initiale, 0 m, 50 (de mercure). Après 6 heures d'effluve, pression $0^{\mathrm{m}}$, 07. Le vase renfermait encole de roxygène ot de l'azote.

Poids du gaz alssobé $\left(\mathrm{A} z^{2}+\mathrm{O}^{5}\right)$ : Crr,016 d'après les volumes; 0,015 d'après le titre acide. Réaction presque totale.

(5) Même mélangè, en présence diune solution étendue de potasse. Volume initial du gaz réduit : $t^{\mathrm{cm}}, 6$. Pression intérieure initiale: $0^{m 7} 764, t=8{ }^{0}, 5$. Durée, 14 heures. Pression finale, (1m09,

Gaz absorbé, $O=0 \mathrm{~g}, 042, \mathrm{~A} Z=0 \mathrm{~s}^{\mathrm{n}}, 0171$; ce qui répond à AZ $0^{2} H=0 r^{r}, 096$. Daprès le titre acide, 0;092.

Autre experience sur $24^{m 3}, 5$. Gaz initial. On a obtenu $\mathrm{AzO}^{3 \mathrm{H}}=0 \mathrm{br}, 038$ d'après les volumes ahsorbés. D'aprës le volume AzO régénépé, 0.037. $\mathrm{Ni} \mathrm{AzH}$, ni AzO2H.

(6) Même mélange, à $80^{\circ}$. Autre vase. Pression initiale des gaz froids, $0 \mathrm{~m} 50$. AzO $\mathrm{O}^{3} \mathrm{H}$ formé : d'après les volumes gazeux, Orr,010; d'après le titre acide, 0,0108.

(7) Melange de 2 volumes d'azote et de 1 volume d'ox. gène, $\mathrm{Az} z^{2}+\mathrm{O}$, en prísence de l'eau. Pression initiale, $0^{\mathrm{m}}, 77, t=8^{\circ}$. Durée : 6 heures. Volume initial réduit, $53^{\mathrm{cm} 3}, 6$. Volume final du gaz réduit, $28^{\mathrm{cm} 3}, 4$.

Diminution: $25^{\mathrm{cm} 3}, 2$, formés de $17^{\mathrm{cm} 3}, 9$ oxygène et $7 \mathrm{~cm}, 2$ azote (mesures). Rapport 5: 2. Tout l'oxygène a disparu. Ni $\mathrm{AzAH}^{3}$, ni $\mathrm{AzO}^{2} \mathrm{H}$

17 bis) Résultat semblable, avec le même mélangre, en presence de la potasse.

(8) Mème mélange. Pression atmosphérique initiale.

(c) En réduisant l'épaisseur de la couche gazeuse, il pourrat en être atrenent bans tous les eas, les décharges discuptres, sous forme d'âncelle ou de plure de feu, ont toujours dé érutées.

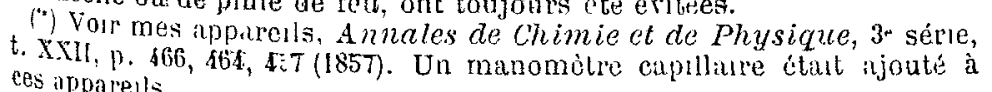
es ippareuls.
Volume initial réduit, $71^{\mathrm{cm} 3}, 3$. Voluménnal, $38^{\mathrm{cm} 3}, 3$. Durée 8 heures. Diminution: $33 \mathrm{~cm}^{3}$. Tout l'oxygène a disparu, soit $0=23^{\mathrm{cm} 3}, 8$ et $\mathrm{A} z=9 \mathrm{~cm} 3.5$. Trace diacide azoteux.

Poids de $\mathrm{AzO}^{3} \mathrm{H}$ : daprès les volumes gazeux absoirbés, ogr053; d'après le volume régénéré de $\mathrm{A} z \mathrm{O}, 0,051$.

(9) Air ordinaire, en présence de la potasse étendue. Pression initiale, $0^{\mathrm{m}}, 768, t=13^{\circ}$. Pression finale, $0^{\mathrm{m}}, 58$. II reste un peu d'oxygène.

$\mathrm{AzO}$ [I obtenu, d'après les volumes gazeux, cwr,057; d'après le titre acide $0,05 \overline{7}$. Ni AzH $\mathrm{N}^{3}$, ni AzO $2 \mathrm{H}$.

(9 bis) Air, en présence do leau. Expénence semblable. Gaz initial réduit, $33^{\mathrm{cm} 3}, 8$. Gaz final, $24 \mathrm{~cm} 3,8$, renfermant encore $0^{\mathrm{cm} 3}$, y d'oxygène. Azote restant, 24'm3,5. D'où $\mathrm{AzO}^{3} \mathrm{~L}$ formé, d'après les volumes gazeux. 0rr.0137. D'après le titre acide, 0,0130 ; d'après $A z O$ régénéré, 0,0136 .

On voit par ces expériences que la combinaison de l'oxygène el de l'azote en présence de la vapeur d'eau, opérée à hasse température par l'effluve, tend à s'accomplir jusquà épuisement de l'oxygène, quelle que soit la proportion relative de l'azote; cette limite n'étant dailleurs atteinte complètement qu'au bout d'un temps thêriquement infini. Dans tous les cas étudiés, il se forme de liacide azotique, sans formation sensible d'ammoniaque ou d'acide azoteux: tant qu'il y a un excès d'oxysène, et même avec un excès d'azote.

Ce résultat pourrait s'expliquer en présence d'un excès d'oxygène, en raison de la tendance de l'oxygrène à former de l'ozone, et de l'incompatibilité entre l'ozone et l'acide azoteux, du moins lorsquils sont maintenus pendant quelque temps en présence de l'eau; ainsi qu'entre l'ozone et l'ammoniaque.

Mais cette explication ne suffit pas pour expliquer l'absence de l'acide azoteux, en présence d'un excès d'azote. H semble dès lors établi que l'acide azotique est, dans tous les: cas, le produit essentiel de la réaction, et que l'azote n'est pas susceptible de le ramener à l'étal d'un oxyde inférieur, dans les conditions de mes expériences.

Cette formation de lacide azotique á peu près exclusive, en présence d'un mélange d'azote, d'oxygène et de vapeur d'eau, a lieu non seulement à la température ordinaire, mais jusqu'à $80^{\circ}$ au moins.

Il convient évidemment de faire des réserves pour les. températures élevées au-dessus du degré où s'opère la dêcomposition pyrogénée de l'acide azotique.

Ajoutons d'ailleurs que la réaction offre le même caractère, depuis la pression atmosphérique normale du début, jusqu'aux très faibles pressions finales d'oxygène, lesquelles répondent á la disparition à peu près totale de ce gaz dans les mélangres.

Troisième partie. - RELATIONS ENTRE la VITESSE DE LA COMBINAISON ET LA PRESSTON DU SYSTEME GAZEUX

En opérant dans les conditions qui viennent d'être signalées, le volume des gaz intérieurs demeure sensiblement constant (sauf les petites différences attribuables au changement de niveau du mercure, dans lespace très petit occupé par ce liquide dans le tube capillaire).

En tenant compte des variations de température de l'ail et de la pression atmospherique, on peut dès lors déduire de la hauteur de la colonne mercurielle (qui définit la pression du gaz expérimenté) le poids absolu d'acide azotique, formé pendant une période quelconque de l'expérience. C'est ainsi qu'ont été obtenus les résultats suivants.

Je commencerai par les mélangres gazeux formés dans les proportions théoriques $\mathrm{A}^{\mathrm{D}}+\mathrm{O}^{5}$; latension de la vapeur deau étant de $0^{m}, 008$ vers $8^{\circ}$, température voisine de celle de la plupart de ces essais. Cette tension est d'ailleurs négligeable dans une premiere approximation; saut vers la fin du phénornène, bien entendu.

Soit d'abord l'expérience (5), exéculée sur un volume: 
gazeux de $64^{\mathrm{m} 3}, 6$ (réduit). Pression initiale: $0^{\mathrm{m}}, 76 ; t=8^{\circ}, 5$. Pression finale: $0 \mathrm{~m}, 08$. Durée, 14 heures. Quantité totale d'acide $\mathrm{AzO}^{3} \mathrm{H}$ formée: $9 \mathrm{~b}^{\mathrm{m} g \mathrm{r}}$.

Au début, l'action s'exerce avec une certaine intensité; elle s'accélère ensuite, par une sorte d'entrainement. Au bout de trois heures et demie (210m11), la pression est tombée à $0{ }^{m}, 34$, un peu moins de moitié. La quantité d'acide formé s'élevait alor's à 50 mgr (52 pour 100 de la totalité); c'està-dire la moitié envıron: soit $0 \mathrm{mgr}, 24$ par minute.

Pendant les 2 heures qui suivent (de $210^{\mathrm{m} m}$ à $330^{\mathrm{m}} \mathrm{n}$ ), la pression tombe à 0 m, 25 . La quantite d'acide formé pendant cet intervalle était de $10 \mathrm{mgr}, 8(11 \mathrm{p}$. $100 \mathrm{du}$ total $)$, soit $0^{\mathrm{m}} \mathrm{rr}, 9$ par minute: un peu moins de moitié de la précédente, pour la même durée et pour une pression réduite de moitie. Pendant les 4 heures suivantes $\left(330^{\mathrm{m}} \mathrm{n}\right.$ à $\left.570^{\mathrm{mm}}\right)$, la pression tombe a $0^{\mathrm{m}}, 156$. J.e poids dacide forme pendant ce nouvel intervalle était de $11 \mathrm{mmr}, 5$, soit 12 pour 100 du total, c'est-ì-dire $0^{\mathrm{mgr}}, 48$ par minute: moitié environ de la période précédente, pour une pression réduite d'un tiers.

La réaction se ralentit ainsi indéfiniment, à mesure que l'état de condensation de la matière diminue. C'est là une conclusion applicable à une multitude de phénomènes climiques. Quil me soit permis de rappeler qu'elle a été inaugurée dans l'étude des réactions gazeuses, par mes expériences sur la formation des éthers, en 1860. Il n'est pas possible d'ailleurs de calculer une loi rigoureuse de ce décroissement durant la formation de lacide azotique d'aprós les chiffres qui précèdent, à cause de l'accélération initiale et de la variation de tension de la vapeur d'eau, en premier lieu. En outre, la vitesse de la réaction est surtout influencée par la variation incessante el irrégulière des tensions électriques, et par suite de l'énergie fournie à chaque instant par la bobine dinduction, dans les conditions présentes de son emploi (*).

La conclusion générale qui précède n'en demeure pas moins certaine.

(10) Voici une autre expérience, exécutée sur un volume de $27^{\mathrm{cm} 3}$, c'est-à-dire à peu près moitié moinảro que les précédentes, avec formation finale de 0 rr, 038 de $\mathrm{AzO} \mathrm{O}^{3} \mathrm{H}$, en présence de KOH étendue.

Pendant les premières 15 minutes, la pression est tombée a $0^{\mathrm{m}}, 68$. Le poids moyen d'acide formé par minute était $0 \mathrm{mgr}, 16$.

Pendant les 30 minutes suivantes, la pression tombe à $0^{\mathrm{m}}, 57$. Poids moyen d'acide formé par minute. $0^{\mathrm{mgr}}, 21$.

Pendant les 30 ininutes suivantes, la pression tombe à $0^{\mathrm{m}}, 42$ Poids moyen d'acide formé par minute, $0 \mathrm{mgr}, 28$.

Ainsi, pendant les 75 premières minutes, la réaction a formé $06^{r}, 017$ de $\mathrm{AzO}^{3} \mathrm{H}$, c'est-à-dire près de moitié du poids total obtenu à la fin; la pression étant réduite a près de moitié.

Pendant les 150 minutes suivantes, la pression tombe à $0^{\mathrm{m}}$ 17. Poids moyen de l'acide formé par minule, $0^{\mathrm{m} g \mathrm{r}}, 095$. Il $\mathrm{y}$ a donc nouvelle réduction do pression de plus de moité; le poids de l'acide azotique formé pendant cette période s'élévant à $0 \mathrm{gr}, 014$.

Pendant les 120 minutes suivantes, la pression est réduite à $0 \mathrm{~m}, 076$ Poids moyen d'acide formé par minute, $0^{\mathrm{mgr}}, 044$.

Pendant les 120 minutes suivantes, la pression se réduit enfin à $0^{m}, 04$. Poids moyen d'acide formé par minute, $0 \mathrm{mgr}, 016$.

On voit ici plus clairement l'accélération initiale, puis la

() Pour arriver à une loi prócıse, Il faudrat, en outre, définur plus nettement l'éparsseur de la couche gazeuse sur laquelle s'excrce l'action de l'elluve, cpassenr qui n'est pas uniforme dans les apparells de verro. Il faudrait aussil définir le mode de distribution de l'action de lefluve dans les diflerentes régions de la masse gazeuse, et spécialement dans la capicilé vide, en partie lemplie d eau, qu sépare le fond du tube intéreur de celui du lube concentrique qui lenveloppe. infin, il corviendrat de fare intervonir la vitesse d'evaporation de lean restituéc. diminution progressive, qui se produit en raison de la diminution de la condensation de la matière gazeuse.

(11) Voici deux expériences, destinées à rendre compte, autant que possible, de linfluence d'un condensateur électrique (bouteille de Leyde) : condition oú l'étincelle modé. ratrice entre les pôles change, comme on sait, de longueur et d'intensité. Ces deux expériences ont été exécutées avec los mêmes tubes, afin d'en rendre les conditions compara. bles, autant que possible. Au contraire, elles ne le sont pas au même degré avec les précédentes, en raison de la diffé. rence des volumes intérienrs et de l'éparsseur de la couche gazeuse interposée. Volume intérieur, $24 \mathrm{~cm}$. Pression intiale, $0^{m}, 50$.

\begin{tabular}{|c|c|c|c|c|c|}
\hline \multirow{3}{*}{\multicolumn{2}{|c|}{ Polds total de $\mathrm{AzO} \mathrm{O}^{3} \mathrm{H}$ formé }} & \multicolumn{2}{|c|}{$\begin{array}{c}\text { SANS CONDENSATEUR } \\
0 \mathrm{gr}, 0 ! 86\end{array}$} & \multicolumn{2}{|c|}{$\begin{array}{l}\text { AVEC CONDENSATELR } \\
0 \mathrm{gr}, 0 \pm 9\end{array}$} \\
\hline & & & Pouls d $\mathrm{AzO}^{3} \mathrm{H}$ & & Polds d'AzOB \\
\hline & & l'ression & par musule. & Pressunt & par minale \\
\hline es 15 mimules & suivantes & $0 \mathrm{~m}, 38$ & $0 \mathrm{mg} .17$ & $0 \mathrm{~m}, 40$ & $r, 19$ \\
\hline es 30 minutes & sulvantes & $0 m, 24$ & $0 \mathrm{~m}, 20$ & $0 \mathrm{~m}, 30$ & $0 \mathrm{mg}, 175$ \\
\hline & survalutes & $0 m, 15$ & $0 \mathrm{mg}, 06$ & $0 \mathrm{~m}, 20$ & $0 \mathrm{mg}, 08$ \\
\hline $60 \mathrm{mi}$ & survantes & $0 m 07$ & $0 m 15,05$ & $0 m, 12$ & $0 \mathrm{mg}, 06$ \\
\hline
\end{tabular}

La combınason avait altent ì peu près la mostié Nême observation an de la limite au bout d'une heure bout d'une heure.

D'après ces observations, l'influence du condensateur électrique employé a été minime; conséquence qu'il ne faudrait pas trop généraliser pour d'autres dispositions.

\section{Quatrième PARTIE. - INFLUEnCe D'Un EXCÉs D'Azote}

Jusqu’à quel point la condensation gazeuse décroissante de la fraction du mélange gazeux, entrant en combinaison et par l'effet de cette combinaison, dans un mélange renfermant en excès de l'un des composants, tel que l'azote, peut-elle être assimilée à la simple raréfaction produite sur le système $\mathrm{A} z^{2}+\mathrm{O}^{5}$, dont les composés entrent intégralement en réaction? C'est ce que font entrevoir les expériences suivantes.

Soient les chiffres obtenus au cours de l'expérience (8), exécutée sur le mélange $\mathrm{Az}^{2}+\mathrm{O}$.

En présence de la potasse, au boul de 8 heures, toutloxygène, ou sensiblement, a disparu, et le volume réduit du gaz final s'est trouvé réduit à $54 \mathrm{p} .100$ de son volume initial. La théorie indique en effet pour la formation exclusive de l'acide azotique dans un semblable melange : 53 pour 100 .

En fait d'ailleurs (*), le poids de cet acide $\mathrm{A} z \mathrm{O}^{3} \mathrm{H}$ formé s'élevaít à $0 \mathrm{mg}, 053$.

Or.pendant la première heure, il s'est formé 0 orr, 017 d'acide, soit le liel's de la quantité totale : $0 \mathrm{mgr}, 3$ environ par minute moyenne. Pendant l'heure suivante, il s'est formé 0sr,012 d'acide : soit $0^{\text {nigr }}, 2$ par minute moyenne.

Pendant les 2 heurcs suivantes, 0war,08 par minute moyemne.

Pendant les 2 heures suivantes, $0^{\mathrm{mgr}, 05}$ par minute moyerne.

Pendant les 2 heures suivantes, $0 \mathrm{mgr}, 02$ par minute moyenne.

En fait, les poids absolus obtenus sont voisins de ceux de l'expérience (10); mais ils représentent une fraction du total: $[0 \mathrm{gr}, 053$ dans l'expérience (8), au lieu de $0 \mathrm{gr}, 038$, dans l'expérience (10)]

L'action parait donc plus lente, en présence d'un excès d'azote, pour une même tension relative de la fraction combinable.

Cependant, les vitesses n'ont pas été réduites á moitié environ, comme il semble qu'il aurait dú résulter de la condensation propre du mélange transformable, dilué à peu près à la moltié de la condensation réalisée en l'absence d'un excès d'azote. 11 semble donc, que les conditions des

*) Par une concidence numérique fortule. 
expériences actuelles autoriseraient à admettre que l'excès d'azote a une certaine influence favorable : ce qui serait conforme aux lois connues de la combinaison chimique, telle que je l'ai observée dans l'étude des réactions éthérées. Mais les conditions actuelles sont trop complexes pour ăutoriser une conclusion définitive.

\section{III. - Thermochimie}

La réaction fondamentale défnie plus haut:

$$
\mathrm{Az}^{2}+\mathrm{O}^{5}+\mathrm{H}^{2} \mathrm{O}+\mathrm{Aq}=2 \mathrm{AzO} \mathrm{O}^{3} \mathrm{H} \text { étendu }
$$

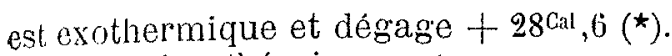

La lóaction théorique entre corps gazeux :

$$
\mathrm{Az}^{2}+\mathrm{O}^{5}+\mathrm{H}^{2} \mathrm{O} \text { gaz }=2 \mathrm{AzO} \mathrm{O}^{3} \mathrm{H} \text { gaz }
$$

légagerait +9 cal, 4 , si l'on admettait que les hydrates dacide azolique gazeux, susceptibles de se former en présence d'un excès de vapeur d'eau. et de subsister dans cet état, fussent entierement dissociés dans l'état gazeux; hypothèse qui jeut itre contestée. Quoi qu'il en soit, on voit, que la formation de l'acide azotique demeurerait en tous cas exothormique.

II resulte de là que l'effluve joue surtout dans la formation directe de l'acide azolique, à la température ordinaire, le rôle de déterminant ou de catalysateur, (dans le langage actuel); sans fournir une énergie consommée au cours de l'accomplissement de la combinaison.

Or, ll en est tout autrement de la combinaison directe de larote el de l'oxygène par l'arc ou par l'étincelle électrique, soil avec production de bioxyde d'azote:

$$
2 \mathrm{Az}+2 \mathrm{O}=9 \mathrm{AzO} \text {, absorberait à frold }-43^{\mathrm{Cal}}, 2 \text {; }
$$

solt avec production de gaz hypoazotique :

$$
2 \mathrm{Az}+2 \mathrm{O}^{2}=2 \mathrm{AzO}^{2} \text {, absolberait }-18 \mathrm{cal}, 5 \text { vers } 200^{\circ},
$$

Az0² étant formé seul;

ou bien, à troid, -3 al ,4;

cette delnière température donnant naissance à un mélange do deux composés inégalement condensés, tels que

$$
\mathrm{AzO}^{2}+\frac{1}{2} \mathrm{Az}^{2} \mathrm{O}^{k}
$$

La réaction serait donc toujours endothermicue au voisinage de la température ordinaire.

Quant à ce qui se passe réellement aux températures de l'arc ou de l'étincelle, il n'est pas possible de l'évaluer avec cerhtude, parce que nous ignorons quelles seraient les valeurs des chaleurs spé ¿ifiques des gaz simples ou composés à ces températures. Si l'on se conforme aux analogies. tirées de la connaissance générale des relations constatées entre les chaleurs spécificues des graz simples el celles des gaz composés, il semble que la chaleur absorbée dans la formation des oxydes de l'azote par leurs éléments doit croltre en valeur absolue avec l'élévation de la température. Il est donc probable que cette formation, telle qu'elle est réalisée en fait par l'étincelle ou l'arc électrique, demeure endothermique : étant accomplie par le concour's des énergres électriques, étrangères au système atomique de ces eléments libres. Son caractère parait dès lors essentiellement dilférent de celui de la formation de lacide azotique par l'uftuve, telle qu'elle est étudiée dans le présent mémoire.

Il en est de même de la formation des oxydes de l'azote, simultanée avec la combustion du carbone, du soufre ou de l'hydrogène; la réaction totale résultant de ces combustions et de la formation accessoire de petites quantites d'oxydes d'azote. Dès lors ce sont ces dernières combustions qui fournmaient l'énergie consommée dans la dernière formation.

(*) Termoch mie. Données el lois numériques, t. II, p. 106.
Le mot même de combustion, appliqué à l'oxydation de l'azote par l'électricité est équivoque; car cette oxydation, accomplie par une énergie étrangère et extérieure au système atomique avec absortion de chaleur, n'est nullement assimilable à la combustion londamentale du carbone, du soufre ou de l'hy drogène. Je ne saurais trop insister, au point de vue de la mécanique chimique, sur cette différence essentrelle qui existe entre la synthèse directe de l'acide azotique, opérée à basse température, ot la formation des oxy les de l'azote aux température-élèvées, avec ou sans concours de l'électricité.

\section{Loi du 15 Juin 1900} SUR LES

\section{DISTRIBUTIONS D'ÉNERGIE}

Nous donnons ì nos lecteurs le texte de cette loi si longtemps attendue, et qui vient de voir le jour. Ils trouveront en même temps le rapport de $M$. Chautemps, sénateur, quicontient quelques observations intéressantes.

Mais nous nous empressons d'annoncer que la librairie Gratier et Rey, à Grenoble, mettra en vente, $\dot{a}$ partlr du $\iota^{\text {or }}$ aout un commentaire de cet te loz dù à la plume de notre collaborateur, M. Bougault, avocat à la Cour d'appel de Lyon.

Ce livre est appelé à préciser la situation noullle que crée la loi ci-jointe a tous les entrepreneurs de distribution d'énergie: le régime de l'autorisation, celun de la concession simple, celui de la concession avec déclaration d'utilité publique; il a pour but d'indiquer dans quelles conditions on doit recourtr a telle uu telle solution; quelles sont les formalutés à remplir, quelle est la situation de l'éclarrage, ses privilèges, l'absence de monopole pour la distribution d"énergie, etc. (*).

RA PPORT fait au nom de la Commission( $\left.{ }^{*}\right)$ chargée d'examiner la proposition de loi, adoplée par la Clambre des Diputés, sar les Distributions d'énergie, par M. Ch.sutemps, sénateur.

Dans sa séance du 27 février I906, la Chambre des Députés a adopté, sur le rapport de $M$. Léon Janet, une proposition de loi concernant les distrubutions d'énergic électrique non destinées à la télégraphie ni à la téléphonıe.

Cette question était pendante devant la Chambre des Députés depuis le I 2 juillet $I 897$, jour où fut déposé le projet de lo1 qu'avait élaboré une Commission présidée par M. Armand Rousseau, conseiller d'Etat (1894), et qui avait été adopté plus tard (1896) par le Conseil d'Etat.

Elle avait donné lieu à un rapport de M. Guillain ( 8 février 1898), puis, sous la législature suivante, de M. André Berthelot.

Les premiers projets de lo1s (rapports Guillain ex Berthelot) s'appliquaient aux divers modes de transport de l'énergie, aux conduites d'eau à haute pression et aux conduites d'air comprimé comme aux conducteurs d'électricité ; celui qui a été finalement adopté par la Chambre des Députés, sur le rapport de $\mathrm{M}$. Léom Janet, ne vise que les distributions d'énergie électrique.

Une autre dissemblance sépare encore le texte adopté par la Chambre des Députés de celui qui était annexé au rapport de M. André Berthelot, c'est que ce dernier s'appliquait seulement aux entreprises ayant pour objet la distribution de l'énergie électrique au public, tandis que le texte présenté à la Chambre des Députés par M. Léon Janet s'applique également aux distributions n'intéressant que les particuliers. Le texte adopté présente enfin, sur le projet Berthelot, cet

(*) Les souscriptions sont reçues dès aujourd'hui à la librairie Gratier et Rey, à Grenoble. Prix de l'ouvrage, 5 francs.

(*) Cette Commission était composée de MM. Berthelor, président; Genet, secrétaire; Folgerrol, Paul Le Rovx, Chactemps, Charles Prevet, Gourant, Pédebidou, Francoz. 